\title{
The DAS28-ESR cutoff value necessary to achieve remission under the new Boolean-based remission criteria in patients receiving tocilizumab
}

\author{
Yasuhiko Hirabayashi • Tomonori Ishii • \\ for the Michinoku Tocilizumab Study Group
}

Received: 29 March 2012 / Revised: 18 August 2012 / Accepted: 9 October 2012 /Published online: 23 October 2012

(C) The Author(s) 2012. This article is published with open access at Springerlink.com

\begin{abstract}
To seek the cutoff value of the 28-joint disease activity score using erythrocyte sedimentation rate (DAS28ESR) that is necessary to achieve remission under the new Boolean-based criteria, we analyzed the data for 285 patients with rheumatoid arthritis registered between May 2008 and November 2009 by the Michinoku Tocilizumab Study Group and observed for 1 year after receiving tocilizumab (TCZ) in real clinical practice. Remission rates under the DAS28-ESR criteria and the Boolean criteria were assessed every 6 months after the first TCZ dose. The DAS28-ESR cutoff value necessary to achieve remission under the new criteria was analyzed by receiver operating characteristic (ROC) analysis. Data were analyzed using last observation carried forward. After 12 months of TCZ use, remission was achieved in 164 patients ( $57.5 \%$ ) by DAS28ESR and 71 patients $(24.9 \%)$ under the new criteria for clinical trials. CRP levels scarcely affected remission rates, and the difference between remission rates defined by DAS28-ESR and by the new criteria was mainly due to patient global assessment (PGA). Improvement of PGA was inversely related to disease duration. ROC analysis revealed that the DAS28-ESR cutoff value necessary to predict remission under the new criteria for clinical trials was 1.54 , with a sensitivity of $88.7 \%$, specificity of $85.5 \%$, positive predictive value of $67.0 \%$, and negative predictive value of $95.8 \%$. A DAS28-ESR cutoff value of 1.54 may be reasonable to predict achievement of remission under the
\end{abstract}

\section{Y. Hirabayashi $(\bowtie)$}

Department of Rheumatology, Hikarigaoka Spellman Hospital,

6-7-1 Higashisendai, Miyagino-ku,

Sendai 983-0833, Japan

e-mail: mr.hirabayashi@gmail.com

T. Ishii

Department of Hematology and Rheumatology,

Tohoku University Hospital,

Sendai, Japan new Boolean-based criteria for clinical trials in patients receiving TCZ.

Keywords Boolean · Criteria · DAS28-ESR · Remission · Tocilizumab

\section{Introduction}

Rheumatoid arthritis (RA) is a chronic inflammatory disease which fluctuates in activity during the course of the disease. To evaluate disease activity at the time of observation, the 28joint disease activity score using erythrocyte sedimentation rate (DAS28-ESR) was developed in 1995 and the cutoff value representing remission was defined as $<2.6$ [1]. However, we now know that multiple joints can remain swollen or tender at this cutoff value. Because several biologics have now become available, achievement of complete clinical remission has become a practical goal and the DAS28-ESR definition of remission has now become unsuited for use in real clinical practice. Therefore, a new definition of remission based on a Boolean approach was approved by the American College of Rheumatology (ACR) and the European League Against Rheumatism (EULAR) [2]. The new criteria for clinical trials define remission to be when tender joint count (TJC), swollen joint count (SJC), CRP (in milligrams per deciliter), and scores on a patient global assessment using a visual analogue scale (PGA-VAS) $(0-10-\mathrm{cm}$ scale) are all $\leq 1$. The same criteria, but excluding CRP, are the new criteria for defining remission in clinical practice.

DAS28-ESR has been used worldwide and contributes considerably to the standardization of evaluation of disease activity. If the DAS28-ESR cutoff value necessary to achieve remission under the new ACR/EULAR Boolean-based criteria is found, it will be possible to reanalyze a lot of the accumulated data based on DAS28-ESR. In this report, we sought this cutoff 
value on the basis of prospectively registered observational data by the Michinoku Tocilizumab Study Group (MTSG) which was organized to evaluate the efficacy and safety of tocilizumab (TCZ) in real clinical practice.

\section{Subjects and methods}

\section{Subjects}

The subjects of this analysis were patients meeting the 1987 revised RA classification criteria from the ACR and who had newly received TCZ following its marketing approval in Japan for use in RA. A total of 285 patients in 34 institutions in the Tohoku area were registered between May 2008 and November 2009 by the MTSG. Their demographics and baseline characteristics prior to treatment are as follows: mean age \pm standard deviation (SD) was $59.6 \pm 13.1$ years; male/female ratio was 21:79; mean disease duration $\pm \mathrm{SD}$ was $10.2 \pm 9.1$ years; the percentage of patients of Steinbrocker's classes I, II, III, and IV was $21,57,22$, and $0 \%$, respectively; Steinbrocker's stages I, II, III, and IV was $10,23,29$, and $38 \%$, respectively; mean $\mathrm{TJC} \pm \mathrm{SD}$ was $6.5 \pm 6.0$; mean $\mathrm{SJC} \pm \mathrm{SD}$ was $5.6 \pm 5.1$; mean $\mathrm{ESR} \pm \mathrm{SD}$ was $50.6 \pm 32.8 \mathrm{~mm} / \mathrm{h}$; mean $\mathrm{CRP} \pm \mathrm{SD}$ was $2.7 \pm$ $2.8 \mathrm{mg} / \mathrm{dl}$; mean PGA-VAS $\pm \mathrm{SD}$ was $5.43 \pm 2.47 \mathrm{~cm}$ (on a 0 10 -cm scale); and mean DAS28-ESR \pm SD was $5.2 \pm 1.3$.

\section{Methods}

This study was not an intervention study and was approved by the Ethics Committee of Tohoku University. The patients had been observed for 1 year after receiving TCZ in real clinical practice. TJC, SJC, PGA-VAS, ESR, and CRP were assessed every 6 months after the first TCZ dose to calculate the remission rates under the DAS28-ESR definition and under the new 2011 ACR/EULAR Boolean-based remission criteria. The DAS28-ESR cutoff value necessary to achieve remission under the new criteria was analyzed by receiver operating characteristic (ROC) analysis. Data were analyzed using last observation carried forward.

\section{Results}

Proportions of patients achieving the criteria or each component of the criteria

In the 285 patients, the 6-month- and 12-month TCZ continuation rates were 87.4 and $81.8 \%$, respectively. After 6 months of TCZ use, remission was achieved by 152 patients $(53.3 \%)$ under the DAS28-ESR definition, by 71 patients (24.9\%) under the new criteria for clinical practice, and by 68 patients $(23.9 \%)$ under the new criteria for clinical trials (Fig. 1). After 12 months, remission was achieved by 164 patients $(57.5 \%)$ under the DAS28-ESR definition, by 72 patients $(25.3 \%)$ under the new criteria for clinical practice, and by 71 patients $(24.9 \%)$ under the new criteria for clinical trials. ESR decreased from $50.6 \pm 32.8 \mathrm{~mm} / \mathrm{h}$ at baseline to $14.2 \pm 21.8$ after 6 months and to $13.9 \pm 21.7$ after 12 months. The mean DAS28-ESR decreased from 5.2 at baseline to 2.7 after 6 months and to 2.6 after 12 months. Next, the number of patients achieving each component of the Boolean-based
Fig. 1 Percentage of patients achieving each component of the Boolean-based remission criteria or achieving remission at 6 months (gray bars) or 12 months (black bars)

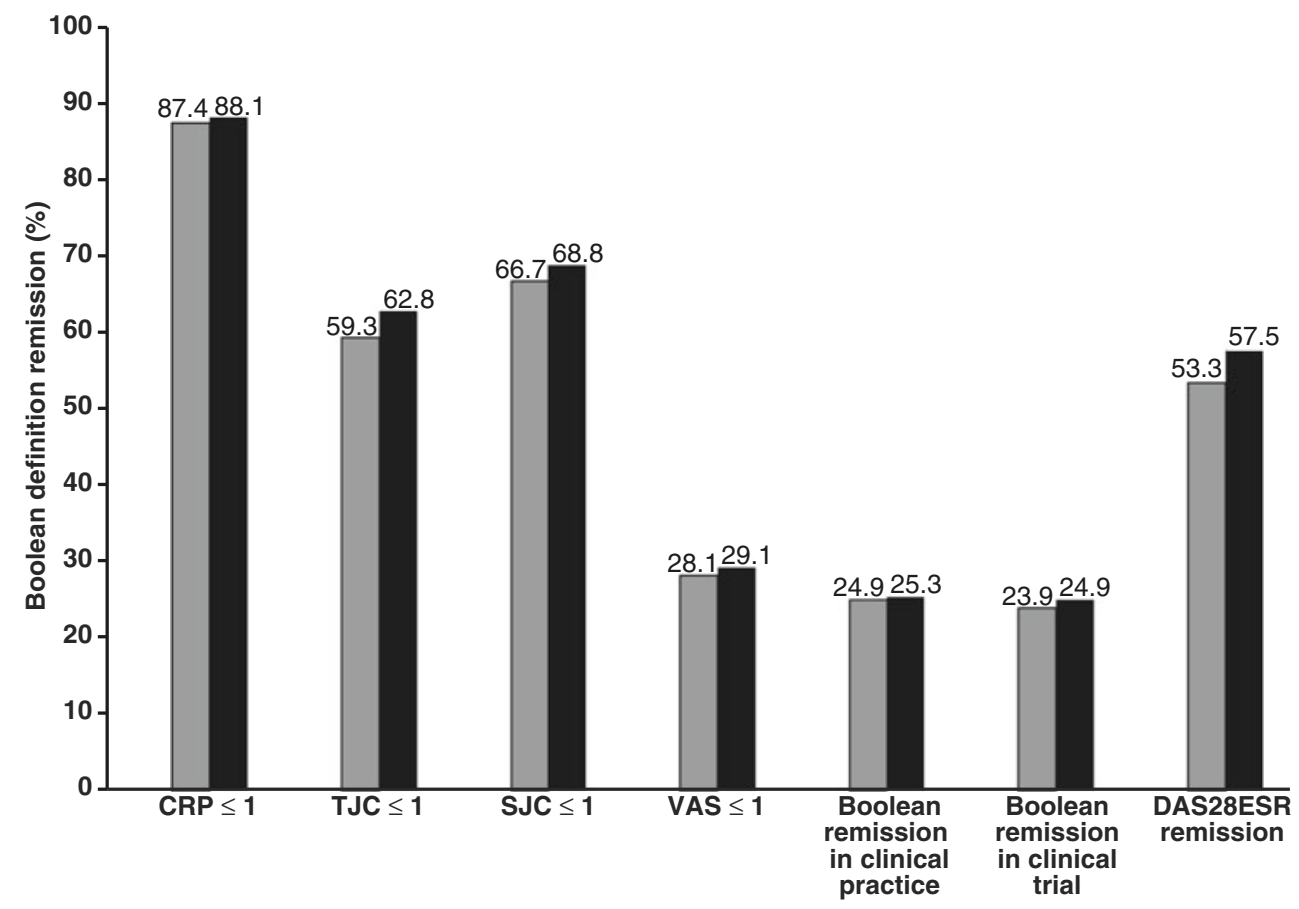


remission criteria was tallied. CRP $\leq 1 \mathrm{mg} / \mathrm{dl}$ was achieved by 249 patients $(87.4 \%)$ at 6 months and 251 patients $(88.1 \%)$ at 12 months. TJC $\leq 1$ was achieved by 169 patients $(59.3 \%)$ at 6 months and 179 patients $(62.8 \%)$ at 12 months. SJC $\leq 1$ was achieved by 190 patients $(66.7 \%)$ at 6 months and 196 patients $(68.8 \%)$ at 12 months. PGA-VAS $\leq 1 \mathrm{~cm}$ was achieved by 80 patients $(28.1 \%)$ at 6 months and 83 patients $(29.1 \%)$ at 12 months.

DAS28-ESR cutoff value necessary to predict achievement of remission under the new criteria

The DAS28-ESR cutoff values necessary to achieve remission under the new criteria for clinical trials and for clinical practice were analyzed by ROC analysis (Fig. 2). The analysis revealed that a value of 1.54 was the most accurate cutoff value for both sets of criteria, with a sensitivity of $88.7 \%$ (trials) and $87.5 \%$ (practice), specificity of $85.5 \%$ (trials) and $85.5 \%$ (practice), positive predictive value of $67.0 \%$ (trials) and $67.0 \%$ (practice), and negative predictive value of $95.8 \%$ (trials) and $95.3 \%$ (practice). The area under the ROC curve was 0.91 in both sets of criteria, indicating high accuracy.

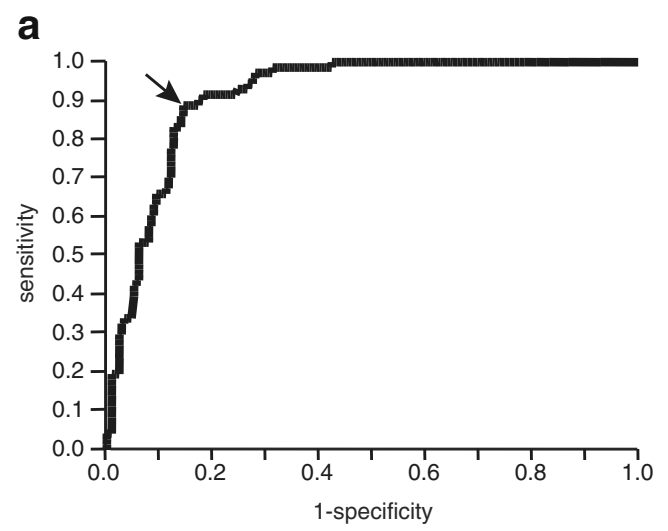

b

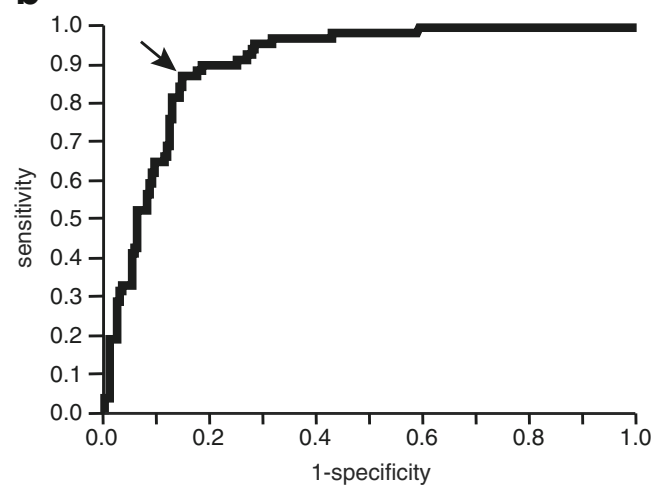

Fig. 2 Receiver operating characteristic curve of DAS28-ESR $<1.54$ for the ACR/EULAR Boolean-based definition of remission in clinical trials (a) and in clinical practice (b). The arrow indicates the point where the value of [sensitivity - $(1-$ specificity $)]$ becomes maximum
Effect of disease duration on achievement of remission

Compared to the negative predictive value, the positive predictive value was not so high. To clarify the reason for this, we compared the proportions of patients with DAS28ESR $<1.54$ achieving each component of the remission criteria in the following two groups: those with disease duration $<10$ years (Group $1 ; n=59$ ) and those with disease duration $\geq 10$ years (group $2 ; n=35$ ) (Fig. 3 ). CRP $\leq 1 \mathrm{mg} / \mathrm{dl}$ was achieved by 59 patients $(100.0 \%)$ in group 1 and 35 patients $(100.0 \%)$ in group 2 . TJC $\leq 1$ was achieved by 58 patients $(98.3 \%)$ in group 1 and 35 patients $(100.0 \%)$ in group 2 . SJC $\leq 1$ was achieved by 56 patients $(94.9 \%)$ in group 1 and 34 patients $(97.1 \%)$ in group 2 . In these three components, there was no significant difference between the two groups. On the other hand, a significant difference was observed in PGA-VAS. PGA-VAS $\leq 1 \mathrm{~cm}$ was achieved by 47 patients $(79.7 \%)$ in group 1 but only 18 patients $(51.4 \%)$ in group 2. Thus, PGA-VAS is the key component which determines the remission rate, and the improvement of PGA-VAS is inversely related to disease duration.

\section{Discussion}

In this study, our results demonstrated a high rate of DAS28ESR remission (53.3\%) after 6 months, which is compatible with the results of our previous study [3]. Even under the new criteria, about $24 \%$ of patients achieved remission. The

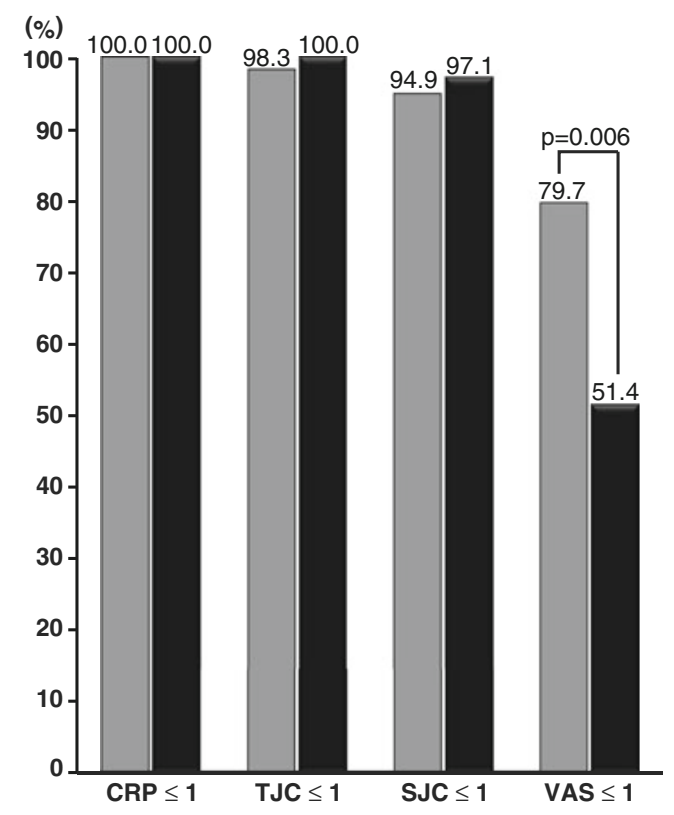

Fig. 3 Percentages of patients with DAS28-ESR $<1.54$ achieving each component of the Boolean-based remission criteria compared by disease duration. Gray bars disease duration $<10$ years, black bars disease duration $\geq 10$ years 
continuation rates at 6 and 12 months were also high. $\mathrm{SJC}<1$ was achieved by $67-69 \%$ of patients indicating that inflammation had mostly subsided. These results are comparable to those of the phase IIIb TAMARA study [4]. There was no significant difference between the remission rate as determined by the new criteria for clinical trials and that for clinical practice $(P=0.923)$. This means that, although TCZ stops CRP gene transcription [5], the remission rate was scarcely affected by CRP. The discrepancy between DAS28-ESR and the new criteria was mainly due to PGA (Fig. 1).

ROC analysis revealed that the DAS28-ESR cutoff point necessary to predict achievement of remission under the new criteria, both for clinical trials and for clinical practice, was DAS28-ESR $<1.54$ (Fig. 2). This also means that this cutoff value is not affected by CRP. Sensitivity and specificity were reasonably high. Negative predictive value was very high (>95\%) and the area under the ROC curve was $>0.9$, indicating high accuracy. Moreover, the cutoff value is clearly below the more stringent DAS28-ESR cutoff point (DAS28-ESR <2.0) examined by the ACR and the EULAR as a candidate definition of remission [2]. Therefore, DAS28-ESR $<1.54$ may be a reasonable cutoff value to predict achievement of remission under the new ACR/EULAR Boolean-based criteria for TCZ. Of course, it is not known whether this value can be used for other antirheumatic drugs until other drugs are studied in the same way because TCZ strongly improves ESR by markedly ameliorating inflammatory anemia [6] and decreasing inflammatory molecules such as fibrinogen [7].

A new definition of remission based on the simplified disease activity index (SDAI) was also approved by the ACR and the EULAR [2]. However, in this study, we could not indicate a DAS28-ESR cutoff value predicting SDAI $<3.3$ because the prospectively registered observational data did not include the physician global assessment. We would like to investigate this when we have a chance.

In general, longer disease duration is associated with more severe joint destruction. Understandably, joint destruction decreases quality of life and increases the points on the Health Assessment Questionnaire (HAQ). This increased HAQ score is the damage-related component of the HAQ (DAM-HAQ) of which improvement is not easy [8,9]. At the damaged joints, motion pain frequently remains even after treatment has resulted in disappearance of inflammation. Therefore, although most patients achieved TJC $\leq 1$ and $\mathrm{SJC} \leq 1$, improvement of PGA-VAS is limited in the patients with longer disease duration (Fig. 3). This means that, due to DAM-HAQ, criteria using PGA-VAS could be inaccurate for evaluating disease activity in patients with longer disease duration. One way to improve the accuracy of assessing remission would be to change the cutoff value of PGA-VAS depending on the disease duration or the degree of joint destruction such as determined by the modified total Sharp score.

Acknowledgments We would like to thank the MTSG investigators, their staff, and their patients for participation in this study. In addition to the authors (Y. Hirabayashi and T. Ishii), MTSG investigators (all in Japan) included the following (in alphabetical order): Masataka Abe (Kitakami Saiseikai Hospital, Kitakami) Hiroshi Abiko (Nihonkai General Hospital, Sakata), Takanobu Ando (Iwate Medical University, Morioka), Noriyuki Chiba (Morioka National Hospital, Morioka), Kojiro Endo (Hoshi General Hospital, Koriyama), Hiroshi Fujii (Tohoku University Hospital, Sendai), Akira Hatakeyama (Tohoku Rosai Hospital, Sendai), Satoshi Hoshi (Apio Riumachi Clinic, Aizuwakamatsu), Kazunobu Ichikawa (Yamagata University Hospital, Yamagata), Yoh Ishiguro (Hirosaki University Hospital, Hirosaki), Eiji Itoi (Tohoku University Hospital, Sendai), Hiroshi Kanazawa (Aomori Prefectural Central Hospital, Aomori), Takashi Kanno (Ohta Nishinouchi Hospital, Koriyama), Ichiro Kato (Tohoku Rosai Hospital, Sendai), Hiroko Kobayashi (Fukushima Medical University Hospital, Fukushima), Takao Kodera (Tohoku KoseiNenkin Hospital, Sendai), Masataka Komagamine (Komagamine Orthopedic and Rheumatology Clinic, Morioka), Atsushi Komatsuda (Akita University Hospital, Akita), Tsuneo Konta (Yamagata University Hospital, Yamagata), Nobuki Maki (Yuri Kumiai General Hospital, Yurihonjo), Keiki Miura (Hiraka General Hospital, Yokote), Takao Miura (Hirosaki Memorial Hospital, Hirosaki), Seiya Miyamoto (Nakadori General Hospital, Akita), Masayuki Miyata (Fukushima Red Cross Hospital, Fukushima), Takatomo Moro (Shirakawa Kosei General Hospital, Shirakawa), Yasuhiko Munakata (Taihaku Sakura Hospital, Sendai), Chihiro Murai (Murai Clinic, Hachinohe), Tai Muryoi (Muryoi Clinic, Shiogama), Haruki Nakano (Yamagata University Hospital, Yamagata), Shigeshi Nakazawa (Nakazawa Sports Clinic, Hachinohe), Ken Ogura (Tohoku Rosai Hospital, Sendai), Hiromasa Ohira (Fukushima Medical University Hospital, Fukushima), Yumiko Oka (Tohoku KoseiNenkin Hospital, Sendai), Hirofumi Okuno (Tohoku University Hospital, Sendai), Naohisa Ouchi (Kitakami Saiseikai Hospital, Kitakami), Yuta Oyama (Nihonkai General Hospital, Sakata), Akira Rikimaru (Kurokawa Hospital, Kurokawa), Koichi Saito (Suminoya Rheumatism \& Orthopedics Clinic, Iwaki), Souichi Saito (Nihonkai General Hospital, Sakata), Hirotake Sakuraba (Hirosaki University Hospital, Hirosaki), Akiko Sasaki (Yamagata University Hospital, Yamagata), Takeshi Sasaki (NTT East Tohoku Hospital, Sendai), Akira Sekiguchi (Tohoku University Hospital, Sendai), Yukio Sekiguchi (NTT East Tohoku Hospital, Sendai), Tadashi Shimamura (Iwate Medical University, Morioka), Kota Sugisaki (Jusendo General Hospital, Koriyama), Mikio Suzuki (Shirakawa Kosei General Hospital, Shirakawa), Yoko Suzuki (Izumi Himawari Clinic, Sendai), Michiaki Takagi (Yamagata University Hospital, Yamagata), Yuichi Takahashi (Yu Family Clinic, Miyagi), Yuya Takakubo (Yamagata University Hospital, Yamagata), Satoshi Takasaki (Yamagata University Hospital, Yamagata), Hiromitsu Takemori (Aomori Prefectural Central Hospital, Aomori), Yukitomo Urata (Seihoku Chuo Hospital, Goshogawara), Yuji Wakai (Hirosaki Memorial Hospital, Hirosaki), Seiji Yamaya (Tohoku University Hospital, Sendai), and Masaaki Yoshida (Yoshida Orthopaedic Clinic, Morioka).

Disclosures Y.H. and T.I. have received speaker's honoraria from Chugai Pharmaceutical Co., Japan.

Open Access This article is distributed under the terms of the Creative Commons Attribution License which permits any use, distribution, and reproduction in any medium, provided the original author(s) and the source are credited. 


\section{References}

1. Prevoo ML, van't Hof MA, Kuper HH, van Leeuwen MA, van de Putte LB, van Riel PL (1995) Modified disease activity scores that include twenty-eight-joint counts. Development and validation in a prospective longitudinal study of patients with rheumatoid arthritis. Arthritis Rheum 38:44-48

2. Felson DT, Smolen JS, Wells G et al (2011) American College of Rheumatology/European League Against Rheumatism provisional definition of remission in rheumatoid arthritis for clinical trials. Arthritis Rheum 63:573-586

3. Hirabayashi Y, Ishii T, Harigae H (2010) Clinical efficacy of tocilizumab in patients with active rheumatoid arthritis in real clinical practice. Rheumatol Int 30:1041-1048

4. Iking-Konert C, Aringer M, Wollenhaupt J et al (2011) Performance of the new 2011 ACR/EULAR remission criteria with tocilizumab using the phase IIIb study TAMARA as an example and their comparison with traditional remission criteria. Ann Rheum Dis 70:1986-1990
5. Ramji DP, Vitelli A, Tronche F, Cortese R, Ciliberto G (1993) The two C/EBP isoforms, IL-6DBP/NF-IL6 and C/EBP delta/NF-IL6 beta, are induced by IL- 6 to promote acute phase gene transcription via different mechanisms. Nucleic Acids Res 21:289-294

6. Emery P, Keystone E, Tony HP, Cantagrel A, van Vollenhoven R, Sanchez A, Alecock E, Lee J, Kremer J (2008) IL-6 receptor inhibition with tocilizumab improves treatment outcomes in patients with rheumatoid arthritis refractory to anti-tumour necrosis factor biologicals: results from a 24-week multicentre randomised placebo-controlled trial. Ann Rheum Dis 68:1516-1523

7. Zhang Z, Fuentes NL, Fuller GM (1995) Characterization of the IL6 responsive elements in the $\gamma$ fibrinogen gene promoter. J Biol Chem 270:24287-24291

8. Smolen JS, Aletaha D, Grisar JC, Stamm TA, Sharp JT (2010) Estimation of a numerical value for joint damage-related physical disability in rheumatoid arthritis clinical trials. Ann Rheum Dis 69:1058-1064

9. Aletaha D, Funovits J, Smolen JS (2011) Physical disability in rheumatoid arthritis is associated with cartilage damage rather than bone destruction. Ann Rheum Dis 70:733-739 